\title{
Non-Full Rank Factorization of Finite Abelian Groups
}

\author{
Khalid Amin \\ Department of Mathematics, College of Science, University of Bahrain, Bahrain, Kingdom of Bahrain \\ Email:kamin35@hotmail.com
}

How to cite this paper: Amin, K. (2017)

Non-Full Rank Factorization of Finite Abelian Groups. Open Journal of Discrete Mathematics, 7, 51-53.

https://doi.org/10.4236/ojdm.2017.72005

Received: November 23, 2016

Accepted: April 14, 2017

Published: April 17, 2017

Copyright (c) 2017 by author and Scientific Research Publishing Inc. This work is licensed under the Creative Commons Attribution International License (CC BY 4.0).

http://creativecommons.org/licenses/by/4.0/

\begin{abstract}
Tilings of $p$-groups are closely associated with error-correcting codes. In [1], M. Dinitz, attempting to generalize full-rank tilings of $\mathbb{Z}_{2}^{n}$ to arbitrary finite abelian groups, was able to show that if $p \geq 5$, then $\mathbb{Z}_{p}^{n}$ admits full-rank tiling and left the case $p=3$, as an open question. The result proved in this paper the settles of the question for the case $p=3$.
\end{abstract}

\section{Keywords}

Factorization of Abelian Groups, Error-Correcting Codes

\section{Introduction}

A factorization of a finite abelian group $G$ is a collection of subsets $A_{1}, \cdots, A_{i}, \cdots, A_{k}$ of $G$ such that each element $g \in G$ can be represented in the form $g=a_{1} \cdots a_{i} \cdots a_{k}$. In this case, we write $G=A_{1}, \cdots, A_{i}, \cdots, A_{k}$ and if each $A_{i}$ contains the identity element $e$ of $G$, we say we have a normalized factorization of $G$.

The notion of factorization of abelian groups arose when G. Hajós [3] found the answer to "Minkowski's conjecture" about lattice tiling of $\mathbb{R}^{n}$ by unit cubes or clusters of unit cubes. The geometric version of "Minkowski's conjecture" can be explained as follows:

A lattice tiling of $\mathbb{R}^{n}$ is a collection $\left\{T_{i}: i \in I\right\}$ of subsets of $\mathbb{R}^{n}$ such that $\bigcup_{i \in I} T_{i}=\mathbb{R}^{n}$ and $\operatorname{int}\left(T_{i}\right) \bigcap \operatorname{int}\left(T_{j}\right)=\varnothing$, if $i \neq j, i, j \in I$. Two unit cubes are called twins if they share a complete $(n-1)$-dimensional face. Minkowski was wondering if there exists a tiling of $\mathbb{R}^{n}$ by unit cubes such that there are no twins! Minkowski's conjecture is usually expressed as follows:

Each lattice tiling of $\mathbb{R}^{n}$ by unit cubes contains twins.

As mentioned above, it was G. Hajós [3] who solved Minkowski' conjecture. 
His answer was in the affirmative, after translating the conjecture into an equivalent conjecture about finite abelian groups. Its group-theoretic equivalence reads as follows:

"If $G$ is a finite abelian group and $G=A_{1}, \cdots, A_{i}, \cdots, A_{k}$ is a normalized factorization of $G$, where each of the subsets $A_{i}$ is of the form $\left\{e, a, a^{2}, \cdots, a^{k}\right\}$, where $k<|a|$; here $|a|$ denotes order of $a$, then at least one of the subsets $A_{i}$ is a subgroup of $G$ ".

Redei [4] generalized Hajos's theorem to read as follows:

"If $G$ is a finite abelian group and $G=A_{1} \cdots A_{i} \cdots A_{k}$ is a normalized factorization of $G$, where each of the subsets $A_{i}$ contains a prime number of elements, then at least one of the subsets $A_{i}$ is a subgroup of $G$ ".

\section{Preliminaries}

A tiling is a special case of normalized factorization in which there are only two subsets, say $A$ and $B$ of a finite abelian groups $G$, such that $G=A B$ is a factorization of $G$.

A tiling of a finite abelian group $G$ is called a full-rank tiling if $G=A B$ implies that $\langle A\rangle=\langle B\rangle=G$, where $\langle A\rangle$ denotes the subgroup generated by $A$. In this case, $A$ and $B$ are called full-rank factors of $G$. Otherwise, it is called a non-full-rank tiling of $G$. As suggested by M. Dinitz [1] and also in that of $O$. Fraser and $B$. Gordon [2], finding answers to certain questions is sometimes easier in one context than in others. In this connection consider the group, $\mathbb{Z}_{p}^{n}$ viewed as a vector space of $n$-tuples $\left(x_{1}, x_{2}, \cdots, x_{n}\right)$ over $\mathbb{Z}_{p}$. Then subspaces correspond to subgroups. Moreover, $\mathbb{Z}_{p}^{n}$ is equipped with a metric, called Hamming distance $d_{H}$, which is defined as follows:

$$
\text { For } \begin{aligned}
x=\left(x_{1}, x_{2}, \cdots, x_{n}\right) \text { and } y & =\left(y_{1}, y_{2}, \cdots, y_{n}\right), \\
d_{H}(x, y) & =\left|\left\{i: 1 \leq i \leq n, x_{i} \neq y_{i}\right\}\right| .
\end{aligned}
$$

With respect to this metric, the sphere $S(x, e)$ with center at $x$ and radius $e$ is the set $S(x, e)=\left\{y: d_{H}(x, y) \leq e\right\}$.

A perfect error-correcting code is a subset $C$ of $\mathbb{Z}_{p}^{n}$ such that $\bigcup_{x \in C} S(x, e)=\mathbb{Z}_{p}^{n}$ and $S(x, e) \bigcap S(y, e)=\varnothing$, if $x \neq y$.

Observe that in the language of tiling, this says that $\mathbb{Z}_{p}^{n}=C S(0, e)$ is a factorization of $\mathbb{Z}_{p}^{n}$ [6].

\section{Factorization and Partition}

Let $G=A B$ be a factorization of a finite Abelian group $G$. Then the sets $\{a B: a \in A\}$ form a partition of $G$. Also, $|G|=|A||B|$, where $|A|$ as before denotes the number of elements of $A$.

\section{Definition}

Let $A$ and $A^{\prime}$ be subsets of $G$. We say that $A$ is replaceable by $A^{\prime}$, if whenever $G=A B$ is a factorization of $G$, then so is $G=A^{\prime} B$.

Redei [4] showed that if $G=A B$ is a factorization of $G$, where $A=\left\{e, a_{1}, a_{2}, \cdots, a_{p-1}\right\}$, and $p$ is a prime, then $A$ is replaceable by $\left\langle a_{i}\right\rangle$, for each $i, 1 \leq i \leq p-1$. 


\section{Definition}

A subset $A$ of $G$ is periodic, if there exists $g \in G, g \neq e$ such that $g A=A$. It is easy to see that if $A$ is periodic, then $A=H C$, where $H$ is a proper subgroup of $G$ [5].

Before we show the aim of this paper, we mention the following observation. If $G=A B$ is a factorization of $G$, then for any $a \in A$, and $b \in B$, then so is $G=a^{-1} A b^{-1} B$, so we may assume all factorizations $G=A B$ are normalized.

\section{Theorem}

Let $G=\mathbb{Z}_{3}^{n}$ and assume $G=A B$ is a factorization of $G$, where $|A|=3$, then either $A$ or $B$ is a non-full-rank factor of $G$.

Proof:

Note that $|G|=3^{n}$. We induct on $n$.

If $n=1$, then $|B|=1$. Thus, $B$ is a non-full-rank factor of $G$.

Let $n>1$ and assume the result is true for all such groups of order less than $3^{n}$.

Let $A=\{e, a, b\}$. Then in $G=A B$, by Rédei [4], $A$ can be replace by $A^{\prime}=\left\{e, a, a^{2}\right\}$.

If $a^{3}=e$, then $A$ is a subgroup of $G$. Thus, $\langle A\rangle \neq G$, so $A$ is a non-fullrank factor of $G$.

If $a^{3} \neq e$, then from $G=\left\{e, a, a^{2}\right\} B$, we get the following partition of $G$ :

$$
G=e B \bigcup a B \bigcup a^{2} B \cdots(*)
$$

from which we get

$$
G=a B \bigcup a^{2} B \bigcup a^{3} B \cdots(* *) .
$$

Comparing $(*)$ with $(* *)$, we obtain $B=a^{3} B$. Thus, $B$ is periodic, from which it follows that $B=H C$, where $H$ is a proper subgroup of $G$. Now, from $G=A B$, we obtain the factorization $G / H=A B / H=(A / H)(B / H)$ of the quotient group $G / H$, which is of order less than $3^{n}$. So, by inductive assumption, either $\langle A H / H\rangle \neq G / H$ or $\langle B H / H\rangle \neq G / H$ from which it follows that either $\langle A\rangle \neq G$ or $\langle B\rangle \neq G$. That is either $A$ or $B$ is a non-full-rank factor of $G$ QED.

\section{References}

[1] Dinitz, M. (2006) Full Rank Tilings of Finite Abelian Groups, SIAM Journal on Discrete Mathematics, 20, 160-170. https://doi.org/10.1137/S0895480104445794

[2] Fraser, O. and Gordon, B. (1977) Solution to a Problem by A.D. Sands. Glasgow Mathematical Journal, 20, 115-117.

[3] Hajós, G. (1949) Sur la factorisation des groupe abeliens, Casopis Pest. Ma. Fys., 74, 157-162.

[4] Rédei, L. (1965) Die neue Theorie der endlihen abelschen und verallgemeinerung des hauptsatze von Hajos. Acta Mathematica Hungarica, 16, 329-373. https://doi.org/10.1007/BF01904843

[5] Sands, A.D. and Szabo, S. (1991) Factorization of Periodic Subset. Acta Mathematica Hungarica, 57, 159-1167. https://doi.org/10.1007/BF01903814

[6] Szabo, S. (2004) Topics in Factorization of Abelian Groups, Birkhauser, Beijing. 
Submit or recommend next manuscript to SCIRP and we will provide best service for you:

Accepting pre-submission inquiries through Email, Facebook, LinkedIn, Twitter, etc. A wide selection of journals (inclusive of 9 subjects, more than 200 journals)

Providing 24-hour high-quality service

User-friendly online submission system

Fair and swift peer-review system

Efficient typesetting and proofreading procedure

Display of the result of downloads and visits, as well as the number of cited articles Maximum dissemination of your research work

Submit your manuscript at: http://papersubmission.scirp.org/

Or contact ojdm@scirp.org 